\title{
Information Encoding and Decoding Using Unidirectionally Coupled Chaotic Semiconductor Lasers Subject to Filtered Optical Feedback
}

\author{
Flavio Ruiz-Oliveras, Miguel C. Soriano, Pere Colet, and Claudio R. Mirasso
}

\begin{abstract}
We present a detailed numerical study of the dynamics of two unidirectionally coupled semiconductor lasers subject to filtered optical feedback. We show that this chaos-based communication scheme allows for an improvement in the decoding of encrypted messages in comparison with the conventional feedback scheme. We found that the performance of the system is optimal when the closed-loop configuration and similar filters are used in the emitter and receiver systems.
\end{abstract}

Index Terms-Chaos, dynamics, optical feedback, semiconductor lasers, synchronization.

\section{INTRODUCTION}

$\mathbf{S}$ YNCHRONIZATION in nonlinear, chaotic systems is a growing field since the appearance of the pioneering work by Pecora and Carroll in 1990 [1]. They showed that two autonomous similar chaotic systems can synchronize to each other if they are properly coupled. However, synchronization of chaotic systems is somehow counterintuitive since the trajectories of two identical chaotic systems started at nearly the same initial points quickly become uncorrelated. For this reason, practical implementations of synchronized nonlinear chaotic systems have drawn much attention since the appearance of the concept of chaos synchronization.

Chaos synchronization was first demonstrated in electronic circuits [1] and later in coupled solid-state lasers [2]. Two semiconductor lasers subject to optical feedback are yet another example of chaos synchronization that can be found when they are unidirectionally coupled in a master-slave configuration [3], [4] or when they are mutually coupled [5]-[7]. Message encoding/decoding via a chaos-pass filtering process was demonstrated in 2000 [8], yielding a very fast growth of the field in

\footnotetext{
Manuscript received December 10, 2008; revised February 09, 2009. Current version published July 01, 2009. This work was supported in part by MEC, Spain, and Feder under Project TEC2006-1009/MIC (PhoDECC), Project TEC2006-28105-E, and Project FIS2004-00953 (CONOCE2), by the EC Project PICASSO through Grant IST-2005-34551, and by CONACYT, Mexico, under Project 46973. The work of M. C . Soriano was supported by the MEC through a "Juan de la Cierva" contract.

F. Ruiz-Oliveras is with the Centro de Investigaciones en Óptica, 37150 León, Guanajuato, Mexico (e-mail: flavio@ cio.mx).

M. C. Soriano, P. Colet, and C. R. Mirasso are with the IFISC (CSIC-UIB) Instituto de Física Interdisicplinar y Sistemas Complejos, E-07122 Palma de Mallorca, Spain (e-mail: miguel@ifisc.uib.es; pere@ifisc.uib.es; claudio@ifisc. uib.es).

Color versions of one or more of the figures in this paper are available online at http://ieeexplore.iee.org.

Digital Object Identifier 10.1109/JQE.2009.2016982
}

the last few years [9]-[12]. Nowadays, mechanically stable integrated sources are being proposed and tested [13]-[15].

To transmit information, the message has to be encoded in the chaotic output of the emitter system. At the receiver side, a system similar to the emitter needs to synchronize mainly to the chaotic output of the emitter, allowing the extraction of the message [8]. The receiver system filters out the message encoded in the transmitted signal, and the information can be extracted by a simple subtraction. Besides the conventional optical feedback scheme, other configurations including optical injection [4], amplified injection [16], amplified optical feedback [13], incoherent optical feedback and injection [17], [18], optoelectronic feedback [19], and multimode lasers [20] [21] have also been investigated.

In this work, we study numerically the possibility of encoding a message using as a carrier the chaotic output of a semiconductor laser subject to filtered optical feedback. The presence of a filter in the feedback loop can strongly modify the dynamical response of the laser [22]-[25]. A major advantage of filtered feedback systems is that they can produce chaotic emission of narrow bandwidth, which could reduce the synchronization degradation due to bandwidth limitations in the communications channel [26] or crosstalk effects. Furthermore, there are additional advantages that motivate the use of semiconductor lasers subject to filtered optical feedback in the context of chaos-based communications. For instance, the fact that the high frequencies are cut favors the synchronization between emitter and receiver [27]. Also, more importantly, the main parameters of the filter in the feedback loop, such as the detuning of the central frequency of the filter with respect to the solitary laser frequency, and the filter bandwidth, can be used as additional keys to improve the security in the communications, as will be shown later.

We focus on a master-slave configuration, i.e., unidirectional coupling, with two possible schemes: 1) open-loop and 2) closed-loop [28]-[30]. In the open-loop scheme, the emitter, i.e., master laser (ML), is subject to filtered optical feedback while the receiver, i.e., slave laser (SL), is not subject to feedback but to the injection of the emitter's output. In the closed-loop scheme, both lasers are subject to filtered optical feedback, and the SL is also subject to the injection of the emitter's output.

\section{RATE-EQuation MODEL}

The system under consideration is schematically represented in Fig. 1. The ML is always subject to optical feedback, while 
a)

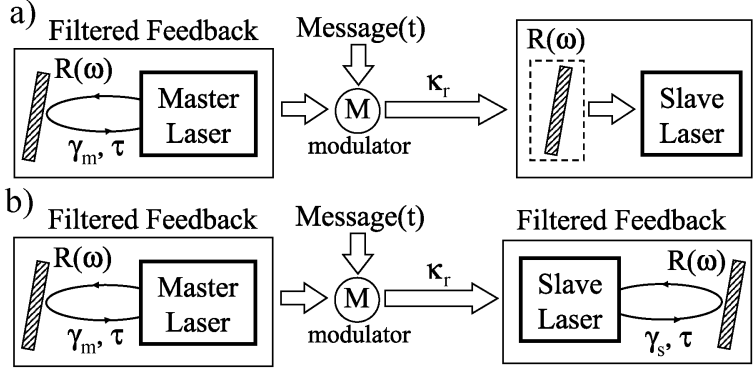

Fig. 1. ML and SL configurations used for all-optical chaos communication systems in a back-to-back configuration: (a) open-loop and (b) closed-loop.

the SL can operate in the open loop [Fig. 1(a)] or in the closedloop configuration [Fig. 1(b)]. In the open-loop configuration, an additional filter can be placed in front of the SL.

Semiconductor lasers subject to optical feedback can be modeled via rate equations. We use a general rate-equations model for single-longitudinal-mode emission. The optical feedback is introduced using the Lang-Kobayashi approach, which takes into account a single reflection in the external cavity. In addition, the external optical feedback is filtered by an external grating, which has a Lorentzian transmission response $R(\omega)$ characterized by a filter half width at half the maximum $\Lambda$ and a central frequency $\omega_{c}$ [22], [23], i.e.,

$$
R(\omega)=\frac{\Lambda}{\Lambda+i\left(\omega_{c}-\omega\right)} .
$$

The equations for the carrier number in the ML (denoted by $m$ ) and SL (denoted by $s$ ) then read

$$
\frac{d N_{m, s}(t)}{d t}=\frac{I}{e}-\frac{N_{m, s}(t)}{\tau_{N}}-G_{m, s}(t) P_{m, s}(t)
$$

and the equations for the evolution of the electric field in the ML read

$$
\begin{aligned}
& \frac{d E_{m}(t)}{d t}=\frac{1+i \alpha}{2}\left[G_{m}(t)-\frac{1}{\tau_{p}}\right] E_{m}(t)+\gamma_{m} F_{m}(t) \\
& \frac{d F_{m}(t)}{d t}=\Lambda_{m} E_{m}(t-\tau) e^{-i \Phi_{m}}+\left(i \Delta_{m}-\Lambda_{m}\right) F_{m}(t) .
\end{aligned}
$$

The evolution of the electric field in the SL in the open-loop scheme read

$$
\begin{aligned}
& \frac{d E_{s}(t)}{d t}=\frac{1+i \alpha}{2}\left[G_{s}(t)-\frac{1}{\tau_{p}}\right] E_{s}(t)+\kappa_{r} F_{p}(t) \\
& \frac{d F_{p}(t)}{d t}=\Lambda_{p} E_{m}(t) e^{-i \Phi_{p}}+\left(i \Delta_{p}-\Lambda_{p}\right) F_{p}(t)
\end{aligned}
$$

and those for the evolution of the electric field in the SL in the closed-loop scheme read

$$
\begin{aligned}
\frac{d E_{s}(t)}{d t}= & \frac{1+i \alpha}{2}\left[G_{s}(t)-\frac{1}{\tau_{p}}\right] E_{s}(t)+\gamma_{s} F_{s}(t) \\
& +\kappa_{r} E_{m}(t) \\
\frac{d F_{s}(t)}{d t}= & \Lambda_{s} E_{s}(t-\tau) e^{-i \Phi_{s}}+\left(i \Delta_{s}-\Lambda_{s}\right) F_{s}(t) .
\end{aligned}
$$

The electric field $F_{m, s}(t)$ is the external optical feedback filtered by a grating, which has a Lorentzian transmission response. $F_{p}(t)$ is the field emitted by the ML that can also be
TABLE I

PARAMETER SET IN THE NUMERICAL SimUlations

\begin{tabular}{|c|c|c|}
\hline Parameter & Description & Value \\
\hline$\alpha$ & linewidth enhancement factor & 5 \\
$\tau_{p}$ & photon lifetime & $2 \mathrm{ps}$ \\
$\tau_{N}$ & carrier lifetime & $2 \mathrm{~ns}$ \\
$g$ & differential gain coefficient & $1.5 \times 10^{-8} \mathrm{ps}^{-1}$ \\
$N_{o}$ & carrier transparency & $1.5 \times 10^{8}$ \\
$s$ & gain compression coefficient & $5 \times 10^{-7}$ \\
$I_{t h}$ & threshold current & $14.7 \mathrm{~mA}$ \\
$I$ & bias current & $30 \mathrm{~mA}$ \\
$\tau$ & feedback delay time & $1 \mathrm{~ns}$ \\
\hline \multicolumn{2}{|c}{} \\
\hline
\end{tabular}

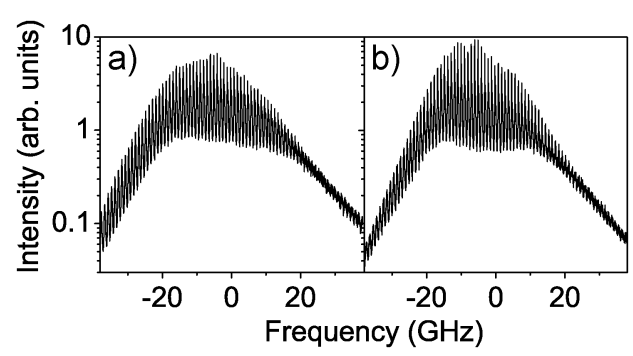

Fig. 2. Optical spectra of a semiconductor laser subject to (a) conventional optical feedback and (b) filtered optical feedback. Parameters: (a) $\gamma_{m}=25 \mathrm{~ns}^{-1}$, (b) $\gamma_{m}=25 \mathrm{~ns}^{-1}$ and $\Lambda_{m} / 2 \pi=15 \mathrm{GHz}$.

filtered by a grating prior to the injection into the SL. For filters of infinite width, $F_{m, s}(t)=E_{m, s}(t-\tau)$ and $F_{p}(t)=E_{m}(t)$. The terms $\kappa_{r} F_{p}(t)$ and $\kappa_{r} E_{m}(t)$ account for the light injected from the ML into the SL, $\kappa_{r}$ is the coupling strength, and the detuning between the ML and SL emission frequencies is initially neglected. For simplicity, the laser parameters are considered to be identical for both devices. $\alpha$ is the linewidth enhancement factor. $\tau_{p}$ is the photon lifetime. $I$ denotes the injected current. $e$ is the electron charge, and $\tau_{N}$ is the carrier lifetime. In the definition of the gain $G_{m, s}(t)=g\left(N_{m, s}(t)-N_{o}\right) /\left(1+s P_{m, s}(t)\right)$, $g$ is the differential gain, $N_{o}$ is the carrier number at transparency, $s$ is the gain compression factor, and $P_{m, s}=\left|E_{m, s}\right|^{2}$ is the optical intensity in terms of the number of photons. $\gamma_{m, s}$ are the feedback strengths. The filters half widths at half the maximum are $\Lambda_{m, s, p}$ and their central frequency detunings, with respect to the free-running emission frequency of the lasers, are $\Delta_{m, s, p}=0 . \tau$ is the external cavity delay and $\Phi_{m, s}=\bmod _{2 \pi}\left(\Delta_{m, s} \tau\right)$ are the phases accumulated by the electric fields in the external cavity round trips. In the long cavity limit, as the one we are considering here, the phases do not play a significant role [11]. Without loss of generality, the flying time between ML and SL is taken as 0 . Table I gives the parameter values that are kept constant through this paper. The relaxation oscillation frequency of the solitary laser is $f_{\mathrm{RO}}=5.9 \mathrm{GHz}$ at $I=30 \mathrm{~mA}$.

\section{SYNCHRONIZATION PROPERTIES}

Under the influence of a filtered optical feedback, the dynamics of the laser can be modified, as can be seen from the optical spectra calculated for different filter widths in Fig. 2. Fig. 2(a) shows the spectrum for conventional optical feedback, and Fig. 2(b) shows the spectrum for the filtered optical feedback case with $\Lambda_{m} / 2 \pi=15 \mathrm{GHz}$. From Fig. 2(b), it can be seen that the spectrum is narrower due to the effect of the filter in the 


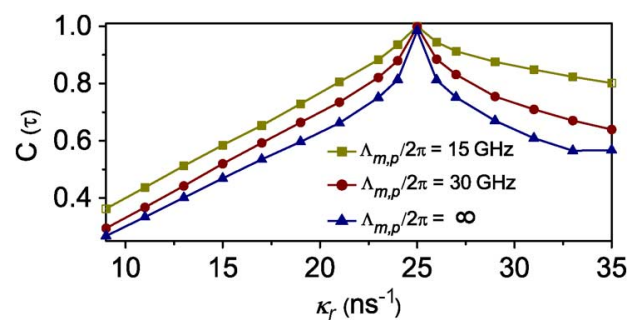

Fig. 3. Correlation coefficient for the achronal solution in the open-loop scheme. $\gamma_{m}=25 \mathrm{~ns}^{-1}$.

external cavity. In this paper, we have chosen filter widths of 15 and $30 \mathrm{GHz}$. The bandwidth of $15 \mathrm{GHz}$, which is around twice the relaxation oscillation frequency, is the minimum filter width for which the dynamics of the laser is still highly chaotic. The bandwidth of $30 \mathrm{GHz}$ is an intermediate value between the minimum bandwidth of $15 \mathrm{GHz}$ and a filter bandwidth of around 60 $\mathrm{GHz}$, whose corresponding dynamics is almost indistinguishable from the one of a laser subject to conventional optical feedback for the parameters considered here.

By narrowing the bandwidth of the dynamics, larger correlations between the ML and SL are expected. In other words, the lasers will synchronize more easily. The cross correlation used to measure the synchronization is defined as

$$
C(t)=\left\langle\left[P_{m}\left(t^{\prime}\right) P_{s}\left(t^{\prime}-t\right)-\bar{P}_{m} \bar{P}_{s}\right]\right\rangle_{t^{\prime}} /\left(\sigma_{P_{m}} \sigma_{P_{s}}\right)
$$

where $\langle\ldots\rangle$ stands for time average, $\bar{P}_{m, s}\left(\sigma_{P_{m, s}}\right)$ are the mean (standard deviation) of the ML and SL output powers, respectively.

We first focus on the open-loop scheme. In this configuration, two solutions are typically found: 1) the isochronous synchronization, where $E_{m}(t)$ is compared with $E_{s}(t)$, and 2) the achronal synchronization, where $E_{m}(t)$ is compared with $E_{s}(t-\tau)$. In filtered optical feedback systems, the achronal synchronization improves when a filter is placed in front of the SL [27]. Note that in this study the current is set to $I=2.04 I_{\mathrm{th}}=30 \mathrm{~mA}$, while in [27] the current is set to $I=1.5 I_{\text {th }}$. Fig. 3 shows the correlation between the ML and the SL in the cases of conventional feedback and filtered feedback with filters of 15 - and $30-\mathrm{GHz}$ widths. As can be seen in Fig. 3, the relation $\gamma_{m}=\kappa_{r}$ [31], [32] must be satisfied in order to obtain the largest possible correlation in the achronal solution. In general, we see that the narrower the filter, the larger the correlations that are obtained.

In the case of the isochronous synchronization and the open-loop configuration, the correlation values are not sufficiently large, when using reasonable values of $\kappa_{r}$, for message encoding and decoding purposes [27], [28]. Only for very large values of the coupling strength is it possible to obtain correlations that can be used for message decoding [27]. Even in this case, large amplitude messages that can compromise the security of the system have to be used [33].

For closed-loop systems, we first analyze the scenario where the ML and SL are identical. Fig. 4 shows the correlation coefficient for different coupling values. As the bandwidth of the filter in the feedback arm is reduced, larger correlations are obtained

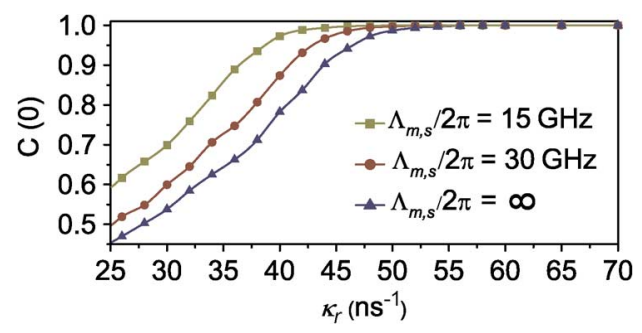

Fig. 4. Correlation coefficient in the closed-loop scheme. $\gamma_{m, s}=25 \mathrm{~ns}^{-1}$.

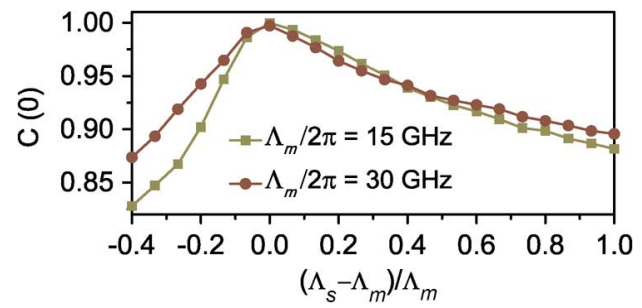

Fig. 5. Correlation coefficient as a function of the SL filter bandwidth mismatch in the closed loop scheme. $\gamma_{m, s}=25 \mathrm{~ns}^{-1}$, and the ML filter bandwidth is kept constant.

for smaller coupling strengths, as it can be seen in Fig. 4. This is due to the combination of the small reduction in the complexity of the dynamics and the filtering of the high frequencies [27].

Let us now consider a parameter mismatch in the bandwidth of the filters used in the ML and SL. While the filter of the SL is varied, the bandwidth of the ML is kept constant. Fig. 5 shows the correlations obtained when the bandwidths of the ML are 15 and $30 \mathrm{GHz}$. We can see that the maximum correlation is always obtained when the feedback loops of both lasers have identical characteristics. We have observed that for larger bandwidths, the correlation coefficient is less sensitive to the filter mismatch. When the bandwidth of the SL filter is smaller than that of the ML, the correlation and synchronization between the two lasers drastically decreases. On the contrary, when the bandwith of the filter in the SL is larger than that of the ML, large correlations can still be obtained, although they slowly decrease as the filter of the SL is increased. We attribute that lack of symmetry to the asymmetries induced by the linewidth enhancement factor.

It is worth mentioning that, by using a filtered optical feedback loop, more variables are involved in the generation of the chaotic carrier. This would allow for a more secure transmission of messages embedded in chaotic carriers. Particularly, if a narrow filter is used in the ML, both lasers need to have almost no mismatch in their filter parameters to have enough correlation to recover a message. This would add an extra difficulty to an eavesdropper trying to recover the message being transmitted, since he would also need to know all the operating characteristics of the filter in the feedback loop of the ML. A more detailed study of the synchronization properties of two unidirectionally coupled lasers subject to filtered optical feedback can be found in [27].

\section{Message EnCoding ANd DeCoding}

The scheme we have chosen to encode the information is chaos modulation (CM), since it is known to perform better 


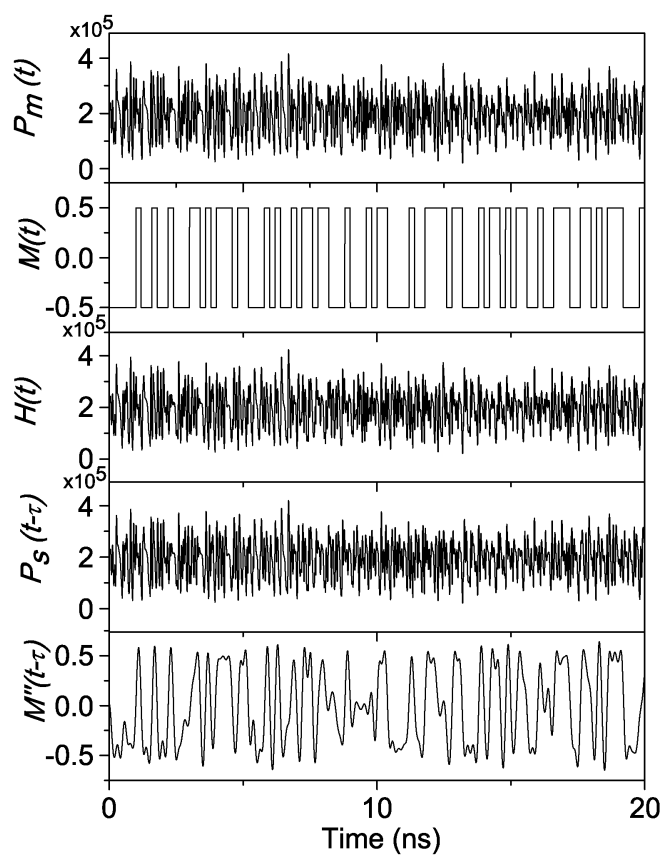

Fig. 6. CM message encoding and decoding in an open-loop scheme where the feedback signal of the ML is filtered with $\Lambda_{m, p} / 2 \pi=15 \mathrm{GHz}$, and $\kappa_{r}=$ $\gamma_{m}=25 \mathrm{~ns}^{-1} \cdot P_{m}(t):$ ML optical intensity, $M(t)$ : transmitted message, $H(t)$ : chaotic carrier with the message, $P_{s}(t-\tau): \tau$-delayed SL optical intensity, and $M^{\prime \prime}(t-\tau): \tau$-delayed recovered message.

than other codification schemes [34]. In this scheme, the message is added by modulating the emitter's chaotic carrier, according to the expression $H(t)=(1-\epsilon M(t)) P_{m}(t)$ [35], where $\epsilon$ is the amplitude of the message encoding, $M(t)$ is the message being transmitted, and $P_{m}(t)$ is the power of the ML. In all of the simulations presented here, $\epsilon=0.04$. The extracted message $M^{\prime}(t)$ is recovered at the receiver side as follows: $M^{\prime}(t)=\left(1-\left(H(t) / P_{s}(t)\right)\right) / \epsilon$, where $P_{s}(t)$ is the power of the SL. The extraction of the message relies on the chaos synchronization, where the SL mainly reproduces the chaotic part of the injected signal $H(t)$, i.e., $P_{s}(t)=P_{m}(t)$ for perfect synchronization. Finally, the extracted message, $M^{\prime}(t)$, is filtered with a fifth-order Butterworth low-pass filter. The cutoff frequency of this filter is set to 1.5 times the message-encoding bit rate. From now on, we denote the decoded message after filtering as $M^{\prime \prime}(t)$. We first study the open-loop scheme and, afterwards, the closed-loop scheme. In the open-loop scheme, we concentrate on the achronal solution, since the isochronous solution yields poor synchronization [27], [28].

\section{A. Open-Loop Configuration}

As we said before, in this study, we focus on the achronal solution when a filter is placed in front of the SL. Fig. 6 shows the decoding of a message when a filter of $15-\mathrm{GHz}$ width is used in the external cavity, for a 5-Gb/s encoding bit rate, and for the optimal condition $\kappa_{r}=\gamma_{m}$ (see Fig. 3).

In Fig. 6, we can see by comparing the original and decoded messages that the information is not completely recovered. In particular, the ML undergoes a chaotic trajectory with higher frequency at the middle of the time series that prevents the SL to properly synchronize to it. Therefore, the decoding of the

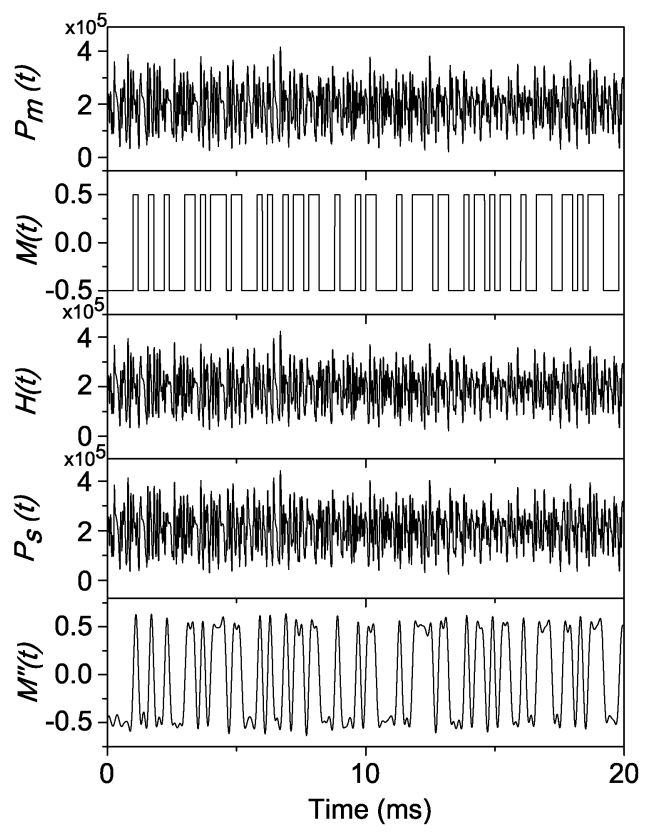

Fig. 7. CM message encoding and decoding in a closed loop scheme where $\Lambda_{m, s} / 2 \pi=15 \mathrm{GHz}, \gamma_{m, s}=25 \mathrm{~ns}^{-1}$, and $\kappa_{r}=70 \mathrm{~ns}^{-1} . P_{m}(t)$ : ML optical intensity, $M(t)$ : transmitted message, $H(t)$ : chaotic carrier with the message, $P_{s}(t)$ : SL optical intensity, and $M^{\prime \prime}(t)$ : recovered message.

message is seriously affected. To quantify the performance of the decoding we calculate the $Q$-factor [35], which is used as a conventional parameter in the evaluation of the performance of the communication systems, given by the expression

$$
Q=\frac{S_{1}-S_{0}}{\left(\sigma_{1}+\sigma_{0}\right)}
$$

where $S_{1}$ and $S_{0}$ are the average optical power of bits "1" and " 0 ," and $\sigma_{1}$ and $\sigma_{0}$ are the corresponding standard deviations.

Within the parameter range considered in our simulations, the performance of the open-loop scheme for decoding a message is very poor. $Q$-factors are as low as 1.17 for conventional feedback and 1.37 for filtered optical feedback with a filter of $15-\mathrm{GHz}$ width. These values of the $Q$-factors yield closed eye diagrams. Hence, the open-loop configuration does not meet the requirements needed to achieve reliable transmission and reception systems [33].

\section{B. Closed-Loop Configuration}

Due to the best performance of the closed-loop scheme [27], we focus on this scheme for the remainder of the paper. Fig. 7 shows the decoding of a $5-\mathrm{Gb} / \mathrm{s}$ encoded message when the bandwidth of the filter is $15 \mathrm{GHz}$ in both ML and SL.

In order to estimate the performance of the closed-loop scheme, we present several examples of eye diagrams and $Q$-factors computed for various configurations. Fig. 8(a) and (b) shows the eye diagrams when a filter of $15-\mathrm{GHz}$ width and a coupling strength of $\kappa_{r}=70 \mathrm{~ns}^{-1}$ are used. For Fig. 8(a), an encoding bit rate of $1 \mathrm{~Gb} / \mathrm{s}$ is considered and for Fig. 8(b) a $5-\mathrm{Gb} / \mathrm{s}$ rate is used. Open and clean eye diagrams, with $Q$-factors as large as 16.28 and 13.39 , respectively, are found. Fig. 8(c) and (d) shows the eye diagrams when a filter of $30 \mathrm{GHz}$ width is placed in the feedback arms. By looking at 


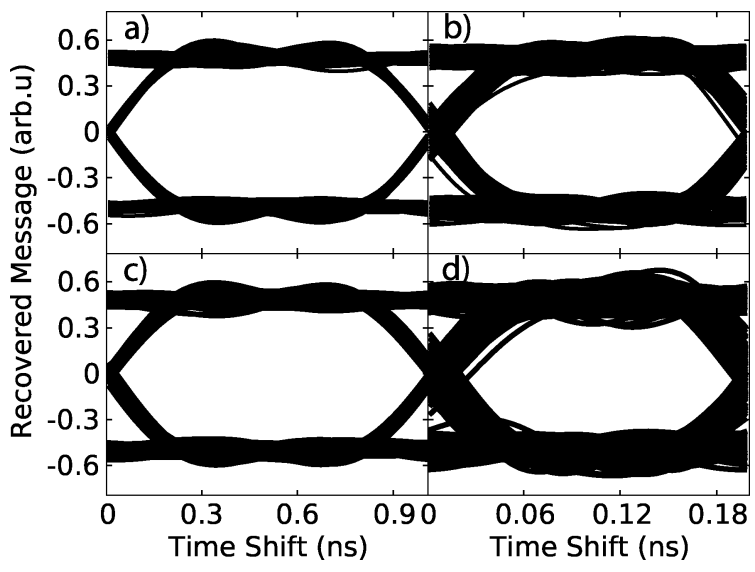

Fig. 8. Eye diagrams with $\kappa_{r}=70 \mathrm{~ns}^{-1}$ and (a) $\Lambda_{m, s} / 2 \pi=15 \mathrm{GHz}$, $1 \mathrm{~Gb} / \mathrm{s}$, (b) $\Lambda_{m, s} / 2 \pi=15 \mathrm{GHz}, 5 \mathrm{~Gb} / \mathrm{s}$, (c) $\Lambda_{m, s} / 2 \pi=30 \mathrm{GHz}, 1 \mathrm{~Gb} / \mathrm{s}$, and (d) $\Lambda_{m, s} / 2 \pi=30 \mathrm{GHz}, 5 \mathrm{~Gb} / \mathrm{s}$.

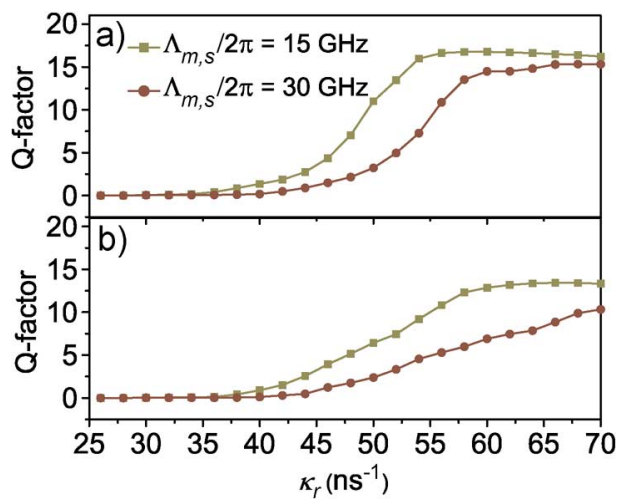

Fig. 9. $Q$-factor as the coupling strength $\kappa_{r}$ is varied for (a) $1-\mathrm{Gb} / \mathrm{s}$ and (b) $5-\mathrm{Gb} / \mathrm{s}$ message-encoding bit rates.

Fig. 8(c) and (d), one can see that the eye diagrams are still very open, with $Q$-factors as large as 15.35 for the $1-\mathrm{Gb} / \mathrm{s}$ encoding bit rate and 10.36 for the $5-\mathrm{Gb} / \mathrm{s}$ rate. However, the quality of the decoding is better for the narrow filter. We have found that the larger the bandwidth of the filter, the larger coupling strength is required to obtain a comparable $Q$-factor. It is worth mentioning that the $Q$-factors for the conventional feedback case are 13.31 for a $1 \mathrm{Gbit} / \mathrm{s}$ encoding bit rate and 9.17 for a $5-\mathrm{Gb} / \mathrm{s}$ rate.

Fig. 9 shows how the $Q$-factor depends on the coupling strength between the ML and SL. Fig. 9(a) is for a 1-Gb/s bit rate, and Fig. 9(b) for a 5-Gb/s bit rate. From these two panels, it can be seen that narrow filters yield better $Q$-factors; moreover, smaller coupling strengths are needed. For conventional optical feedback, the values of the coupling strength needed to obtain comparable $Q$-factors are slightly larger than those needed for the filter of $30-\mathrm{GHz}$ width. If we keep on increasing the coupling strength, the $Q$-factor degrades, i.e., there is an optimum coupling strength where the largest $Q$-factor is obtained [33]. We have observed that the synchronization error increases as well when the coupling strength is larger than this optimum value, making it more difficult to extract the message.

Now, we check the influence of a mismatch in the filter bandwidths. Fig. 10 shows the $Q$-factor when a parameter mismatch in the bandwidth of the filters is introduced. In

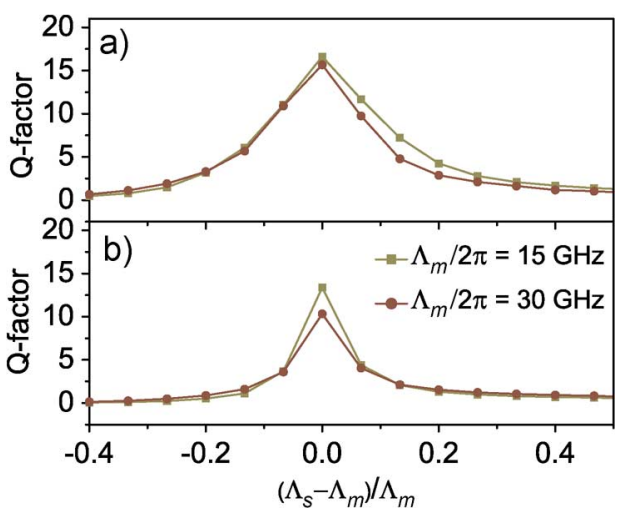

Fig. 10. $Q$-factor as a function of the SL filter bandwidth mismatch, with $\kappa_{r}=$ $70 \mathrm{~ns}^{-1}$, for (a) $1-\mathrm{Gb} / \mathrm{s}$ and (b) $5-\mathrm{Gb} / \mathrm{s}$ message-encoding bit rates.

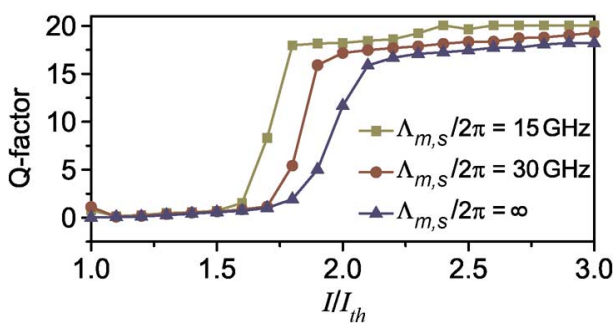

Fig. 11. $Q$-factor versus bias current in the closed-loop configuration for a constant coupling strength $\kappa_{r}=60 \mathrm{~ns}^{-1}$ and an encoding bit rate of $1 \mathrm{~Gb} / \mathrm{s}$.

Fig. 10(a) and (b) the bandwidths of the ML are kept constant to 15 and $30 \mathrm{GHz}$, respectively, while the bandwidth of the SL is scanned. In Fig. 10(a), we use a $1-\mathrm{Gb} / \mathrm{s}$ bit rate and, in Fig. 10(b), a 5-Gb/s bit rate is used. From these panels, it can be seen that a mismatch between the filters degrades significantly the $Q$-factor, and a good message recovery is then almost impossible. The $x$-axis in Fig. 10 has been renormalized to ease the comparison between the two filters. For a filter of $15-\mathrm{GHz}$ width, the $Q$-factor drops below 5 when $\left|\Lambda_{m}-\Lambda_{s}\right|=2 \mathrm{GHz}$ for the $1-\mathrm{Gb} / \mathrm{s}$ rate, and $\left|\Lambda_{m}-\Lambda_{s}\right|=0.9 \mathrm{GHz}$ for the $5-\mathrm{Gb} / \mathrm{s}$ rate. For a filter of $30-\mathrm{GHz}$ width, we can see that the $Q$-factor drops below 5 when $\left|\Lambda_{m}-\Lambda_{s}\right|=4 \mathrm{GHz}$ for the 1-Gb/s rate, and $\left|\Lambda_{m}-\Lambda_{s}\right|=1.8 \mathrm{GHz}$ for the $5-\mathrm{Gb} / \mathrm{s}$ rate. In general, the use of a narrower filter in the ML makes it more difficult for an eavesdropper to find a matching filter in the receiver.

In Fig. 10, we see that a mismatch in the bandwidths of ML and SL can break the message decoding so that the filter bandwidth can be used as an additional key in chaos-based communications. Besides the filter bandwidth, the detuning between the solitary laser frequency and the filter central frequency $\left(\Delta_{m}\right)$ can also be used as an additional secure key. We have checked that values of $\Delta_{m}$ in a range from -10 to $10 \mathrm{GHz}$ do not significantly change the ML dynamics. Hence, any value of $\Delta_{m}$ in the previous range can be used as a secure key.

We end this section by showing the influence of the bias current on the message-decoding performance. In Fig. 11, we present the results for conventional optical feedback and filtered optical feedback, with filters of $15-$ and $30-\mathrm{GHz}$ widths. We have chosen an intermediate value of the coupling strength, $\kappa_{r}=60 \mathrm{~ns}^{-1}$. Fig. 11 shows that the $Q$-factor is larger for larger injection currents. This can be explained in terms of the 
complexity of the dynamics of the ML. It has been shown that the complexity decreases at large currents as a consequence of the gain compression coefficient [36].

\section{CONCLUSION}

We have studied two unidirectionally coupled semiconductor lasers that are driven into a chaotic regime by means of a filtered optical feedback loop. First, we have shown that these lasers can synchronize provided that the filter parameters are matched in the ML and SL. Second, we have shown that the encoding and decoding of a message embedded in a chaotic carrier, generated by a filtered optical system, is feasible.

Our results show that the decoding of encrypted messages yields larger $Q$-factors when a closed-loop scheme is used. The open-loop scheme would be preferred for a preliminary practical implementation since it is known to be mechanically more stable. In this scheme, the achronal solution provides identical synchronization when a filter, similar to the one in the ML, is placed in front of the SL. However, a good message decoding with the achronal solution is, in practice, very difficult.

The synchronization properties of filtered optical feedback systems are similar to the ones found in conventional optical feedback schemes. However, the bandwidth reduction, larger $Q$-factors, and the requirement of smaller coupling strengths make this new scheme very attractive for practical applications.

Finally, we have reported on the influence of a parameter mismatch in the filters of the ML and SL. The $Q$-factors decrease rapidly when the filters are different. This decrease is directly related to a degradation of the synchronization with the mismatch. A filter in the feedback loop of the ML adds an extra degree of difficulty for an eavesdropper trying to extract the encoded information. Actually, it is very demanding to extract the bandwidth of the filter from the time series. We are aware that other alternatives to enhance the security of the communication system can be used, but the system we propose is still a very interesting all-optical option that can be even integrated in compact sources if necessary.

\section{ACKNOWLEDGMENT}

The authors would like to thank Prof. A. N. Pisarchik for helpful discussions.

\section{REFERENCES}

[1] L. M. Pecora and T. L. Carroll, "Synchronization in chaotic systems," Phys. Rev. Lett., vol. 64, pp. 821-824, 1990.

[2] R. Roy and K. S. Thornburg, Jr., "Experimental synchronization of chaotic lasers," Phys. Rev. Lett., vol. 72, pp. 2009-2012, 1994.

[3] C. R. Mirasso, P. Colet, and P. García-Fernández, "Synchronization of chaotic semiconductor lasers: Application to encoded communications," IEEE Photon. Technol. Lett., vol. 8, no. 3, pp. 299-301, Mar. 1996.

[4] V. Annovazzi-Lodi, S. Donati, and A. Scire, "Synchronization of chaotic injected-laser systems and its application to optical cryptography," IEEE J. Quantum Electron., vol. 32, no. 9, pp. 953-959, Sep. 1996.

[5] T. Heil, I. Fischer, W. Elsasser, J. Mulet, and C. R. Mirasso, "Chaos synchronization and spontaneous symmetry breaking in symmetrically delayed coupled semiconductor lasers," Phys. Rev. Lett., vol. 86, pp. 795-798, 2001.
[6] N. Gross, W. Kinzel, I. Kanter, M. Rosenbluh, and L. Khaykovich, "Synchronization of mutually versus unidirectionally coupled chaotic semiconductor lasers," Opt. Commun., vol. 267, pp. 464-468, 2006.

[7] I. Fischer, R. Vicente, J. M. Buldú, M. Peil, C. R. Mirasso, M C. Torrent, and J. García-Ojalvo, "Zero-lag long-range synchronization via dynamical relaying," Phys. Rev. Lett., vol. 97, pp. 123902(1)-123902(4), 2006.

[8] I. Fischer, Y. Liu, and P. Davis, "Synchronization of chaotic semiconductor laser dynamics on subnanosecond time scales and its potential for chaos communication," Phys. Rev. A, vol. 62, pp. 011801(1)-011801(4), 2000.

[9] S. Donati and C. R. Mirasso, "Feature section on optical chaos and applications to cryptography," IEEE. J. Quantum Electron., vol. 38, no. 9, pp. 1138-1140, Sep. 2002

[10] L. Larger and J. P. Goedgebuer, "Cryptography using optical chaos," C. R. Phys., vol. 5, pp. 609-681, 2004

[11] A. Uchida, F. Rogister, J. Garcia-Ojalvo, and R. Roy, "Synchronization and communication with chaotic optical systems," Prog. Opt., vol. 48, pp. 203-341, 2005.

[12] A. Argyris, D. Syvridis, L. Larger, V. Annovazzi-Lodi, P. Colet, I. Fischer, J. García-Ojalvo, C. R. Mirasso, L. Pesquera, and K. A. Shore, "Chaos-based communications at high bit rates using commercial fiberoptic links," Nature, vol. 438, pp. 343-346, 2005.

[13] T. Pérez, M. Radziunas, H.-J. Wünsche, C. R. Mirasso, and F. Henneberger, "Synchronization properties of two coupled multisection semiconductor lasers emitting chaotic light," IEEE Photon. Technol. Lett., vol. 18, no. 20, pp. 2135-2137, Oct. 2006.

[14] M. Yousefi, Y. Barbarin, S. Beri, E. A. J. M. Bente, M. K. Smit, R Nötzel, and D. Lenstra, "New role for nonlinear dynamics and chaos in integrated semiconductor laser technology," Phys. Rev. Lett, vol. 98 , pp. 044101(1)-044101(4), 2006.

[15] A. Argyris, M. Hamacher, K. E. Chlouverakis, A. Bogris, and D. Syvridis, "Photonic integrated device for chaos applications in communications," Phys. Rev. Lett., vol. 100, pp. 194101(1)-194101(4), 2008.

[16] A. Argyris, D. Kanakidis, A. Bogris, and D. Syvridis, "Experimental evaluation of an open-loop all-optical chaotic communication system," IEEE J. Sel. Topics Quantum Electron., vol. 10, no. 4, pp. 927-935, Jul./Aug. 2004

[17] F. Rogister, A. Locquet, D. Pieroux, M. Sciamanna, O. Deparis, P. Megret, and M. Blondel, "Secure communication scheme using chaotic laser diodes subject to incoherent optical feedback and incoherent optical injection," Opt. Lett., vol. 26, no. 19, pp. 1486-1488, 2001.

[18] D. Sukow, K. Blackburn, A. Spain, K. Babcock, L. Bennett, and A. Gavrielides, "Experimental synchronization of chaos in diode lasers with polarization-rotated feedback and injection," Opt. Lett., vol. 29 , no. 20, pp. 2393-2395, 2004

[19] N. Gastaud, S. Poinsot, L. Larger, J. M. Merolla, M. Hanna, J. P. Goedgebuer, and E. Malassenet, "Electro-optical chaos for multi-10 gb/s optical transmissions," Electon. Lett., vol. 40, no. 14, pp. 898-899, 2004.

[20] C. R. Mirasso, M. Kolesik, M. Matus, J. K. White, and J. V. Moloney, "Synchronization and multimode dynamics of mutually coupled semiconductor lasers," Phys. Rev. A, vol. 65, pp. 013805(1)-013805(4), 2001.

[21] J. M. Buldú, J. García-Ojalvo, and M. C. Torrent, "Multimode synchronization and communication using unidirectionally coupled semiconductor lasers," IEEE J. Quantum Electron., vol. 40, no. 6, pp. 640-650, Jun. 2004.

[22] M. Yousefi and D. Lenstra, "Dynamical behavior of a semiconductor laser with filtered external optical feedback," IEEE J. Quantum Electron., vol. 35, no. 6, pp. 970-976, Jun. 1999.

[23] A. P. A. Fischer, O. K. Andersen, M. Yousefi, S. Stolte, and D. Lenstra, "Experimental and theoretical study of filtered optical feedback in a semiconductor laser," IEEE J. Quantum Electron., vol. 36, no. 3, pp. 375-384, Mar. 2000.

[24] A. P. A. Fischer, M. Yousefi, D. Lenstra, M. W. Carter, and G. Vemuri, "Filtered optical feedback induced frequency dynamics in semiconductor lasers," Phys. Rev. Lett., vol. 92, pp. 023901(1)-023901(4), 2004.

[25] K. Green and B. Krauskopf, "Mode structure of a semiconductor laser subject to filtered optical feedback," Opt. Commun., vol. 258, no. 2, pp. 243-255, 2006

[26] M. Kim, C. Sramek, A. Uchida, and R. Roy, "Synchronization of unidirectionally coupled Mackey-Glass analog circuits with frequency bandwidth limitations," Phys. Rev. E, vol. 74, pp. 016211(1)-016211(4), 2006. 
[27] M. C. Soriano, F. Ruiz-Oliveras, P. Colet, and C. R. Mirasso, "Synchronization properties of coupled semiconductor lasers subject to filtered optical feedback," Phys. Rev. E, vol. 78, pp. 046218(1)-046218(8), 2008.

[28] R. Vicente, T. Pérez, and C. R. Mirasso, "Open versus closed loop performance of synchronized chaotic external-cavity semiconductor lasers," IEEE J. Quantum Electron., vol. 38, no. 9, pp. 1197-1204, Sep. 2002.

[29] A. Locquet, C. Masoller, and C. R. Mirasso, "Synchronization regimes of optical-feedback-induced chaos in unidirectionally coupled semiconductor lasers," Phys. Rev. E, vol. 65, pp. 056205(1)-056205(12), 2002.

[30] A. Murakami and J. Ohtsubo, "Synchronization of feedback-induced chaos in semiconductor lasers by optical injection," Phys. Rev. A, vol. 65, pp. 033826(1)-033826(7), 2002.

[31] V. Ahlers, U. Parlitz, and W. Lauterborn, "Hyperchaotic dynamics and synchronization of external-cavity semiconductor lasers," Phys. Rev. E, vol. 58, pp. 7208-7213, 1998.

[32] C. Masoller, "Anticipation in the synchronization of chaotic semiconductor lasers with optical feedback," Phys. Rev. Lett., vol. 86, pp. 2782-2785, 2001.

[33] M. C. Soriano, P. Colet, and C. R. Mirasso, "Security implications of open and closed loop receivers in all-optical chaos-based communications," IEEE Photon. Technol. Lett., vol. 21, no. 7, pp. 426-428, Apr. 2009.

[34] O. Rosso, R. Vicente, and C. Mirasso, "Encryption test of pseudoaleatory messages embedded on chaotic laser signals: An information theory approach," Phys. Lett. A, vol. 372, no. 7, pp. 1018-1023, 2008.

[35] D. Kanakidis, A. Argyris, and D. Syvridis, "Performance characterization of high-bit-rate optical chaotic communication systems in a back-to-back configuration," J. Lightw. Technol., vol. 21, no. 3, pp. 750-758, Mar. 2003.

[36] R. Vicente, J. Daudén, P. Colet, and R. Toral, "Analysis and characterization of the hyperchaos generated by a semiconductor laser subject to a delayed feedback loop," IEEE J. Quantum Electron., vol. 41, no. 4, pp. 541-548, Apr. 2005

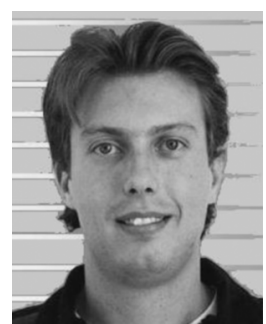

Flavio Ruiz-Oliveras was born in Mexico City, Mexico, in 1976. He received the degree in mechanical engineering from the University of Missouri-Rolla in 2002 and the M.Sc. degree in optics from the Centro de Investigaciones en Optica, A.C., Leon, Mexico, in 2006, where he is currently working toward the Ph.D. degree.

His research interests include nonlinear dynamics and synchronization of chaotic lasers.

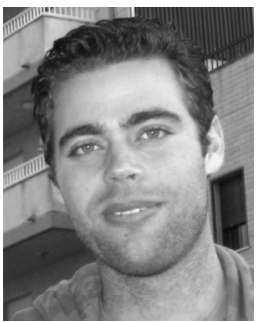

Miguel C. Soriano was born in Benicarlo, Spain, in 1979. He received the degree in telecommunications engineering from the Technical University of Catalonia, Catalonia, Spain, in 2002 and the Ph.D. degree in engineering from the Free University of Brussels, Brussels, Belgium, in 2006.

Since January 2008, he has held a "Juan de la Cierva" scientific contract with the Instituto de Física Interdisciplinar y Sistemas Complejos, Palma de Mallorca, Spain. His main research interests include optical feedback, theoretical modeling, and synchronization properties of semiconductor lasers.

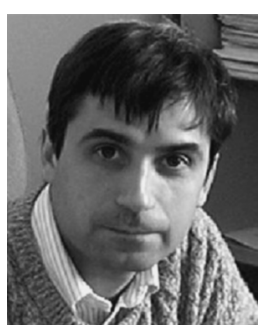

Pere Colet was born in Vilafranca del Penedes, Barcelona, Spain, on April 21, 1964. He received the M.Sc. degree from the Universitat de Barcelona, Barcelona, in 1987 and the Ph.D. degree from the Universitat de les Illes Balears, Palma de Mallorca, Spain, in 1991, both in physics.

In 1991, he became a Teaching Assistant with the Departament de Fisica, Universitat de les Illes Balears. From September 1991 to February 1993 and from April to September 1994, he was a Postdoctoral Fulbright Fellow with the School of Physics, Georgia Institute of Technology, Atlanta. In October 1994, he joined the Departament de Fisica, Universitat de les Illes Balears. Since May 1995, he has held a permanent research position with the Spanish Consejo Superior de Investigaciones Científicas. He has coauthored 60 journal papers as well as 20 other scientific publications. His research interests include fluctuations and nonlinear dynamics of semiconductor lasers, synchronization of chaotic lasers and encoded communications, synchronization of coupled nonlinear oscillators, pattern formation and quantum fluctuations in nonlinear optical cavities, and dynamics of localized structures.

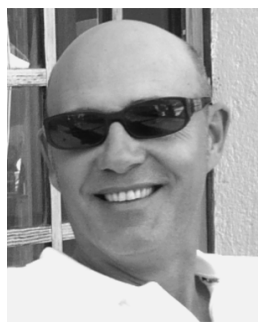

Claudio R. Mirasso was born in Buenos Aires, Argentina, in 1960. He received the M.Sc. and Ph.D. degrees from the Universidad Nacional de La Plata, Buenos Aires, Argentina, in 1984 and 1989, respectively, both in physics.

After several postdoctoral positions in Spain and The Netherlands, he became an Associate Professor with the Physics Department, Universiteit de les Illes Bolears (UIB), in 1996. He has authored or coauthored over 140 publications including about 110 journal papers. His research interests include instabilities in semiconductor lasers, synchronization, and control of chaotic semiconductor lasers, dynamics and applications of delayed coupled semiconductor lasers, and applications of nonlinear dynamics. 